\title{
Anti-Spin Control for Marine Propulsion Systems
}

\author{
Øyvind N. Smogeli ${ }^{1}$, Jostein Hansen ${ }^{2}$, Asgeir J. Sørensen ${ }^{1}$ and Tor Arne Johansen ${ }^{2}$
}

\begin{abstract}
An anti-spin controller for marine propulsion systems in rough seas is developed. From measurements of motor torque and propeller shaft speed, an observer providing an accurate estimate of the propeller load torque is used to calculate an estimate of the torque loss. A monitoring algorithm utilizing the estimated torque loss detects ventilation incidents, and activates the anti-spin control action. When a ventilation situation is detected, the anti-spin control action will reduce the propeller shaft speed to some optimal value, using a combined power/torque controller. The ultimate goal is to minimize the effect of ventilation, and hence increase the thrust production, limit the transients in the power system and reduce the mechanical wear and tear of the propulsion system components. Simulations are provided to validate the performance of the control scheme.
\end{abstract}

\section{INTRODUCTION}

Presently, dynamic positioning (DP) systems have limitations in rough seas. The reasons for this are limitations in the thrust capability and the available power, and reduced performance of the control system due to thrust losses and unmodelled nonlinearities. The most severe thrust loss effects that may be experienced are due to ventilation, which in this work is used to describe air suction to a submerged propeller and in-and-out-of water effects. Ventilation leads to an abrupt and large loss of propeller thrust and load torque [1]. This is challenging for the local thruster controller, which is trying to fulfill the high-level control commands from the DP system. The effects of ventilation are loss of thrust, excessive wear and tear of mechanical components and undesired power transients. It is typically induced by large vessel motions and large waves encountered during harsh weather conditions, and is frequently experienced by e.g. tunnel thrusters and main propellers on offshore supply vessels and shuttle tankers.

In order to counteract the problem of ventilation, the concept of anti-spin thruster control was introduced in [2]. Model tests and simulations of a marine thruster showed the feasibility of increasing thrust production, reducing wear and tear on the mechanical propulsion system and limiting transients in the power system during severe thrust loss incidents by means of controlling the thruster spin. The anti-spin concept was divided in three: Detection, Switching

1 Ø. N. Smogeli and A. J. Sørensen are with Department of Marine Technology, Norwegian University of Science and Technology, NO-7491 Trondheim, Norway. E-mail: [oyvind.smogeli, asgeir.sorensen]@marin.ntnu.no.

2 J. Hansen and T. A. Johansen are with Department of Engineering Cybernetics, Norwegian University of Science and Technology, NO-7491 Trondheim, Norway. E-mail: [jostein.hansen, tor.arne.johansen]@itk.ntnu.no. and Control. However, no explicit control scheme for a general operational setting was presented.

This paper provides an anti-spin control scheme that improves all three parts of the concept, utilizing recent results in thruster control. There are many similarities to car wheel anti-spin control [3], which has served as motivation for the research presented here. The ventilation detection scheme, which triggers the anti-spin control action, is implemented by monitoring an estimate of the propeller torque loss. The estimated torque loss is based on a propeller load torque observer [4]. The torque loss estimate is a convenient variable for detecting the ventilation situations, as it gives explicit and instantaneous information on the thruster load condition. In this work the switching algorithm is by a large part bypassed, as the thruster controller presented here is valid for all load conditions. This simplifies the overall control scheme, and removes any concerns about ensuring bumpless transfer between the various controllers. The torque loss estimate is also used as a basis for the antispin control action.

This work is a continuation of the research on propulsion control, where contributions have been made by for example [5], [6] and the references therein. The local thruster controller used in this work is a combined power/torque control scheme. Power and torque thruster control in marine propulsion systems were first introduced by [7], and recently refined to the combined power/torque control scheme by [4].

\section{THRUSTER MODELLING}

The thruster is modelled as an electric motor, a shaft with friction and a hydrodynamically loaded propeller. The rotational dynamics are described by the following equations:

$$
\begin{aligned}
\dot{Q}_{m} & =\frac{1}{T_{m}}\left(Q_{c}-Q_{m}\right), \\
J \dot{\omega} & =Q_{m}-Q_{p}-K_{\omega} \omega, \\
Q_{p} & =f_{Q}(\theta, \xi),
\end{aligned}
$$

where $T_{m}$ is the motor time constant, $Q_{c}$ is the commanded torque, $Q_{m}$ is the motor torque, $J$ is the rotational inertia of the propeller including added mass, shaft, gears and motor, $K_{\omega}$ is a linear friction coefficient, $\omega$ is the rotational speed of the propeller in $\mathrm{rad} / \mathrm{s}$, and $Q_{p}$ is the propeller load torque. The load torque is modelled as a general function $f_{Q}$ of fixed thruster parameters $\theta$ (i.e. propeller diameter, position, number of propeller blades, pitch ratio, propeller blade expanded-area ratio) and variables $\xi$ (i.e. shaft speed, 
submergence). The actually produced propeller thrust $T_{p}$ is modelled in a similar manner as:

$$
T_{p}=f_{T}(\theta, \xi) .
$$

The nominal thrust $T_{n}$ and torque $Q_{n}$ are typically found from open-water tests with a deeply submerged thruster, expressed by the nominal thrust and torque coefficients $K_{T 0}$ and $K_{Q 0}$ or other equivalent mappings [8]:

$$
\begin{aligned}
T_{n} & =\operatorname{sgn}(n) K_{T 0} \rho D^{4} n^{2}, \\
Q_{n} & =\operatorname{sgn}(n) K_{Q 0} \rho D^{5} n^{2} .
\end{aligned}
$$

Here, $n$ is the propeller shaft speed in rps, $D$ is the propeller diameter, and $\rho$ is the density of water. Thrust losses may be expressed by the thrust and torque reduction coefficients $\beta_{T}$ and $\beta_{Q}$, which express the ratio of actual to nominal thrust and torque [1]:

$$
\beta_{T}=\frac{T_{p}}{T_{n}}=\frac{f_{T}(\theta, \xi)}{T_{n}}, \quad \beta_{Q}=\frac{Q_{p}}{Q_{n}}=\frac{f_{Q}(\theta, \xi)}{Q_{n}} .
$$

In [2] experiments with a ducted propeller in a cavitation tunnel were performed to investigate the effect of ventilation. Fig. 1 shows the nondimensional load torque versus relative submergence $h / R$ and relative shaft speed $n / n_{\max }$, where $h$ is the propeller shaft submergence, $R$ the propeller radius and $n_{\max }$ the maximum shaft speed of the propeller. Based on these results, a generic loss model capturing the main characteristics of ventilation may be formulated. Submergence larger than $h / R \approx 1.5$ should give the conventional quadratic thrust and torque curves as in (3). Decreasing submergence should lead to gradually decreasing thrust and torque for low propeller loading (loss of effective disc area), and a sharp loss of thrust and torque for high propeller loading (air suction). The loss factors $\beta_{T}$ and $\beta_{Q}$ are therefore formulated as functions of the relative submergence and the relative shaft speed, see Fig. 4 later in the text. From this, it is clear that a highly loaded

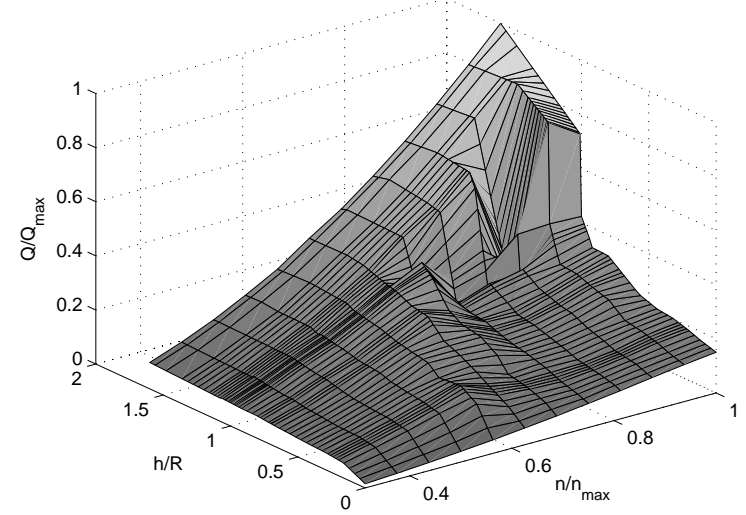

Fig. 1. Experimental results showing nondimensional propeller load torque as a function of relative submergence $h / R$ and relative shaft speed $n / n_{\max }$. thruster operating close to the surface may experience a sudden drop of thrust and torque as ventilation starts. Other loss effects that may be encountered are losses due to inline and cross-flow velocity fluctuations, thruster-thruster interaction and the Coanda effect. However, for sudden and large load fluctuations giving mechanical wear and tear and large power fluctuations, ventilation is the most important loss effect.

\section{LOSS ESTIMATION AND VENTILATION DETECTION}

\section{A. Propeller load torque observer}

An estimate of the propeller load torque is essential for torque loss estimation and ventilation detection. The propeller load torque observer using feedback from motor torque and shaft speed presented in [4] is shortly summarized in the following. Based on (1), the propeller shaft control plant model is written as:

$$
\begin{aligned}
J \dot{\omega} & =Q_{m}-Q_{p}-K_{\omega} \omega, \\
\dot{Q}_{p} & =-\frac{1}{T_{Q}} Q_{p}+w_{Q}, \\
y & =\omega,
\end{aligned}
$$

where the propeller load torque $Q_{p}$ has been modelled as a first order Markov process with time constant $T_{Q}$ driven by white noise $w_{Q}$. With the motor torque $Q_{m}$ as input $u$ and the shaft speed $\omega$ as the measured output $y$, the observer equations for the estimated propeller load torque $\hat{Q}_{p}$ are written as:

$$
\begin{aligned}
\dot{\hat{\omega}} & =\frac{1}{J}\left(-\hat{Q}_{p}-K_{\omega} \hat{\omega}+u\right)+k_{1}(y-\hat{y}), \\
\dot{\hat{Q}}_{p} & =-\frac{1}{T_{Q}} \hat{Q}_{p}+k_{2}(y-\hat{y}), \\
\hat{y} & =\hat{\omega} .
\end{aligned}
$$

The equilibrium point of the observer error dynamics is globally exponentially stable (GES) if $w_{Q}=0$ and the observer gains $k_{1}$ and $k_{2}$ are chosen according to [4]:

$$
k_{1}>-K_{\omega} / J, \quad k_{2}<\left(K_{\omega}+k_{1} J\right) / T_{Q} .
$$

If $w_{Q}$ is a Gaussian white noise process, uniform ultimately boundedness (UUB) follows.

\section{B. Torque loss calculation}

An estimate of the torque loss factor $\beta_{Q}$ may be calculated based on the estimated propeller load torque $\hat{Q}_{p}$ from (6). For DP operation the expected nominal load torque $Q_{n 0}$ is given from (3) by feedback from the propeller shaft speed $n$ as:

$$
Q_{n 0}=\operatorname{sgn}(n) K_{Q 0} \rho D^{5} n^{2} .
$$

The estimated torque loss with respect to the nominal torque expected from the measured shaft speed is then:

$$
\hat{\beta}_{Q}=\alpha_{b}(n)+\left(1-\alpha_{b}(n)\right) \frac{\hat{Q}_{p}}{Q_{n 0}} .
$$


$\alpha_{b}(n)$ is a weighting function of the type:

$$
\alpha(z)=e^{-k|p z|^{r}} \quad \text { for } \quad z \in \mathbb{R},
$$

where $k, p$ and $r$ are positive tuning gains. The weighting function is needed because the estimate otherwise would be singular for zero shaft speed [4].

\section{Ventilation detection}

The thrust loss detection is based on the torque loss estimate $\hat{\beta}_{Q}$. By defining limits for beginning and termination of the ventilation, $\beta_{v, \text { on }}$ and $\beta_{v, o f f}$, a detection signal may be generated by monitoring $\hat{\beta}_{Q}$. This approach is intuitive and robust, and may also be combined with the motor torque detection algorithm in [2]. The loss detection sets the detection flag $\zeta$ to high or low, i.e. $\zeta \in\{0,1\}$. For a single ventilation incident $\zeta$ will have the following evolution, with $t_{1}<t_{2}<t_{3}$ :

$$
\begin{array}{ll}
t_{1}: & \hat{\beta}_{Q} \geq \beta_{v, \text { on }} \Rightarrow \quad \zeta=0, \quad \text { (no ventilation), } \\
t_{2}: & \hat{\beta}_{Q}<\beta_{v, \text { on }} \Rightarrow \zeta=1, \quad \text { (ventilation), } \\
t_{3}: & \hat{\beta}_{Q} \geq \beta_{v, \text { off }} \Rightarrow \zeta=0, \quad \text { (no ventilation). }
\end{array}
$$

See also Fig. 2 later in the text.

\section{THRUSTER CONTROL}

\section{A. Conventional thruster control}

The three thruster control schemes; shaft speed (11), torque (12) and power control (13), are shortly summarized:

$$
\begin{aligned}
n_{d} & =\operatorname{sgn}\left(T_{d}\right) \sqrt{\frac{\left|T_{d}\right|}{\rho D^{4} K_{T 0}}}, \\
Q_{c n} & =f\left(n-n_{d}\right), \\
Q_{c q} & =Q_{d}=\frac{K_{Q 0}}{K_{T 0}} D T_{d}, \\
Q_{c p} & =\frac{P_{d}}{2 \pi|n|}=\frac{Q_{d} 2 \pi n_{d}}{2 \pi|n|} \\
& =\frac{K_{Q 0}}{\sqrt{\rho} D K_{T 0}^{3 / 2}} \frac{\operatorname{sgn}\left(T_{d}\right)\left|T_{d}\right|^{3 / 2}}{|n|}, \quad n \neq 0 .
\end{aligned}
$$

For details, see [7] and [4]. In shaft speed control, shaft speed feedback $n$ from the thruster and the desired shaft speed $n_{d}$ calculated from the desired thrust $T_{d}$ is used to set the commanded motor torque $Q_{c n}$ by for example a PID algorithm $f\left(n-n_{d}\right)$ (11). In torque control the commanded motor torque $Q_{c q}$ is found by a direct mapping from the desired thrust $T_{d}$ (12). In power control the desired power $P_{d}$ is found from the desired torque (12) and the desired shaft speed (11), and the commanded motor torque $Q_{c p}$ (13) is calculated from $P_{d}$ using feedback from the shaft speed. The relationships (3) have been used to relate the desired thrust, torque, shaft speed and power to the nominal thrust and torque coefficients $K_{Q 0}$ and $K_{T 0}$.

\section{B. Combined power/torque thruster control}

The combined power/torque controller presented in [4] is formulated as:

$$
Q_{c c}=\alpha_{c}(n) Q_{c q}+\left(1-\alpha_{c}(n)\right) Q_{c p},
$$

where $Q_{c c}$ is the commanded motor torque, $\alpha_{c}(n)$ is a weight function of the type (10), and $Q_{c q}$ and $Q_{c p}$ are given by (12) and (13). The shape of $\alpha_{c}(n)$ defines the dominant regimes of the two control schemes, and can be used to tune the controller according to user specifications. The combined controller was shown to have smooth behavior with respect to $n$ as long as $r>1$ in the function $\alpha_{c}(n)$, and hence removes the singularity for zero shaft speed found in the power controller. The performance of the combined controller (14) was compared to the conventional shaft speed PID controller (11) in [4] by simulations of a ducted propulsor in the aft ship of a supply vessel in moderate seas. It was shown that the combined controller in absence of ventilation leads to less fluctuations in power and load torque, and also gives a more appropriate thrust production. The combined controller therefore is the preferred controller in normal conditions.

In extreme conditions however, ventilation incidents lead to severe deterioration of the performance of the combined controller, resulting in propeller spin and motor racing. This can be compared to loss of friction for a car wheel, resulting in wheel spin unless the motor torque is rapidly decreased. Propeller spin is an inherent problem also for the torque controller (12) and power controller (13), and motivates the introduction of anti-spin thruster control.

\section{ANTI-SPIN CONTROL}

The anti-spin control action is triggered by the ventilation detection algorithm. The basic concept is to lower the shaft speed once a ventilation situation is detected, i.e. $\zeta: 0 \rightarrow 1$, and increase the shaft speed to normal when the loss situation is considered over, i.e. $\zeta: 1 \rightarrow 0$. The primary anti-spin control action is to modify the controller torque output with a modification factor $\gamma$, giving the following anti-spin controller:

$$
Q_{c a s}=\gamma Q_{c c}=\gamma\left(\alpha_{c}(n) Q_{c q}+\left(1-\alpha_{c}(n)\right) Q_{c p}\right),
$$

where $Q_{c a s}$ is the anti-spin commanded torque. The modification factor would naturally be chosen in the interval $\gamma \in[0,1]$.

At a steady state ventilation condition, the propeller load torque $Q_{p}$, motor torque $Q_{m}$, and commanded torque $Q_{c a s}$ are equal. Using $\gamma=\hat{\beta}_{Q}$, and assuming that the shaft speed $n \neq 0$ and $|n|$ large enough to yield $\alpha_{b}(n) \approx 0$ such that $\hat{\beta}_{Q} \approx \hat{Q}_{p} / Q_{n 0}$ and $Q_{n 0} \neq 0$, the following relationship is established from (9) and (15):

$$
\begin{aligned}
Q_{p} & =Q_{m}=Q_{c a s}=\gamma Q_{c c}=\frac{\hat{Q}_{p}}{Q_{n 0}} Q_{c c} \\
& =\frac{\hat{Q}_{p}}{\operatorname{sgn}(n) K_{Q 0} \rho D^{5} n^{2}} Q_{c c} .
\end{aligned}
$$


If it is further assumed that the estimated propeller torque is identically equal to the actual propeller torque, $\hat{Q}_{p}=Q_{p}$, and that the sign of $n$ and $Q_{c c}$ are equal, then the shaft speed $n$ will be given as:

$$
n=\operatorname{sgn}\left(Q_{c c}\right) \sqrt{\frac{\left|Q_{c c}\right|}{K_{Q 0} \rho D^{5}}} .
$$

In order to investigate the resulting shaft speed, a pure torque and pure power controller are substituted for the combined controller in (17). If assuming that $n$ is small enough to give $\alpha_{c}(n) \approx 1$ such that $Q_{c c} \approx Q_{c q}$, the combined controller becomes a torque controller. The shaft speed in (17) is then from (12) given as:

$$
\begin{aligned}
n & =\operatorname{sgn}\left(Q_{c q}\right) \sqrt{\frac{\left|Q_{c q}\right|}{K_{Q 0} \rho D^{5}}}=\operatorname{sgn}\left(T_{d}\right) \sqrt{\frac{\frac{K_{Q 0}}{K_{T 0}} D\left|T_{d}\right|}{K_{Q 0} \rho D^{5}}} \\
& =\operatorname{sgn}\left(T_{d}\right) \sqrt{\frac{\left|T_{d}\right|}{\rho D^{4} K_{T 0}}} .
\end{aligned}
$$

This is exactly the same as the desired shaft speed $n_{d}$ given by the shaft speed feedback controller (11). If instead assuming that $n$ is large enough to give $\alpha_{c}(n) \approx 0$ such that $Q_{c c} \approx Q_{c p}$, the combined controller becomes a power controller. The shaft speed in (17) is then from (13) given as:

$$
\begin{gathered}
\operatorname{sgn}(n) n^{2}=\frac{\operatorname{sgn}\left(Q_{c p}\right)\left|Q_{c p}\right|}{K_{Q 0} \rho D^{5}} \\
=\frac{1}{K_{Q 0} \rho D^{5}} \operatorname{sgn}\left(T_{d}\right) \frac{K_{Q 0}}{\sqrt{\rho} D K_{T 0}^{3 / 2}} \frac{\left|T_{d}\right|^{3 / 2}}{|n|}, \\
\Downarrow \\
n=\frac{\operatorname{sgn}\left(T_{d}\right)\left|T_{d}\right|^{1 / 2}}{\rho^{1 / 2} D^{2} K_{T 0}^{1 / 2}}=\operatorname{sgn}\left(T_{d}\right) \sqrt{\frac{\left|T_{d}\right|}{\rho D^{4} K_{T 0}}} .
\end{gathered}
$$

This is again the desired shaft speed given by (11). It is therefore assumed that the combined controller (14) as used in (15) will give the same result, as it is a linear combination of the torque and power controllers. If requiring $Q_{c q}=Q_{c p}$ from (12) and (13), the resulting condition is (11), substantiating the assumption. Simulations further confirm this. Using $\gamma=\hat{\beta}_{Q}$ in (15) therefore leads to the same steady-state shaft speed as the shaft speed controller in (11). The following modification factor is proposed as primary anti-spin control action:

$$
\gamma=\left\{\begin{array}{cc}
1 & \text { for } \quad \zeta=0 \\
\hat{\beta}_{Q} & \text { for } \quad \zeta=1
\end{array}\right.
$$

The transition between 1 and $\hat{\beta}_{Q}$ at ventilation detection and termination is done by requiring a rate limit $\kappa$, i.e. $|\partial \gamma / \partial t|<\kappa$, where $\kappa$ typically would be of magnitude 1. When the detection flag changes from 1 to 0 , and the commanded torque again is used directly as motor input, the power control part of the combined controller will assure that no significant power transients occur. In this paper the transient performance of the controller will be validated by simulations.

An additional reduction in shaft speed will be desired during a ventilation incident, calling for a secondary antispin control action. This may be done by modifying the thrust reference during ventilation, since the primary antispin control action assures that the shaft speed corresponding to (11) is kept. The desired shaft speed during ventilation $n_{a s}$ will be a thruster specific parameter which ideally should give the maximum achievable thrust during ventilation. This corresponds to $n / n_{\max } \approx 0.55$ in Fig. 1 and $n / n_{\max } \approx 0.4$ in Fig. 4 , where the two propellers are different. For thrust optimal control, it is a reasonable assumption that $n_{a s}$ may be modelled as a slowly-varying parameter and adapted according to an optimization criterion based on the estimated torque loss. Other optimization criteria may include the power peaks and the torque oscillations. This is subject to further research. The shaft speed $n_{d}$ corresponding to the nominal thrust reference $T_{d}$ should not be changed to $n_{a s}$ instantaneously, as this will lead to undesired transients. It is therefore proposed to add a low-pass filter and rate limiting algorithm to the change from $n_{d}$ to $n_{a s}$ at ventilation detection and from $n_{a s}$ to $n_{d}$ at ventilation termination. The output desired shaft speed from this algorithm is termed $n_{\text {das }}$. The modified thrust reference $T_{d a s}$, which constitutes the secondary anti-spin control action, is given as:

$$
T_{d a s}=\left\{\begin{array}{cc}
T_{d} & \text { for } \quad \zeta=0, \\
T_{n}\left(n_{d a s}\right) & \text { for } \quad \zeta=1,
\end{array}\right.
$$

where $T_{n}\left(n_{d a s}\right)$ is given by (3). The motivation for using the primary anti-spin action in (15) in stead of a conventional shaft speed controller to assure that the secondary anti-spin action in (22) is fulfilled, i.e. $n \rightarrow n_{a s}$ during ventilation, is twofold. Firstly, there is no need to switch between the combined controller and shaft speed controller when ventilation is detected, reducing the problem of ensuring bumpless transfer between the various controllers. Secondly, the combined controller gives smoother control action with rapidly changing inputs, reducing wear and tear and limiting power transients.

A simplified illustration of a ventilation incident with detection and anti-spin control action is sketched in Fig. 2. The figure shows how the torque modification $\gamma$ and the anti-spin shaft speed $n_{\text {das }}$ evolves with the ventilation incident, including detection and transition. Fig. 3 shows a block diagram of the resulting controller, where also a torque limiting function has been included to avoid commanding excessive motor power or torque [7].

\section{Simulation RESUlts}

Simulations of a heavily ventilating propeller were performed to validate the performance of the anti-spin controller given by (15) and (22). The simulated thruster was a ducted propeller of diameter $D=4 m$ installed in the aft ship of a typical supply vessel with main particulars 


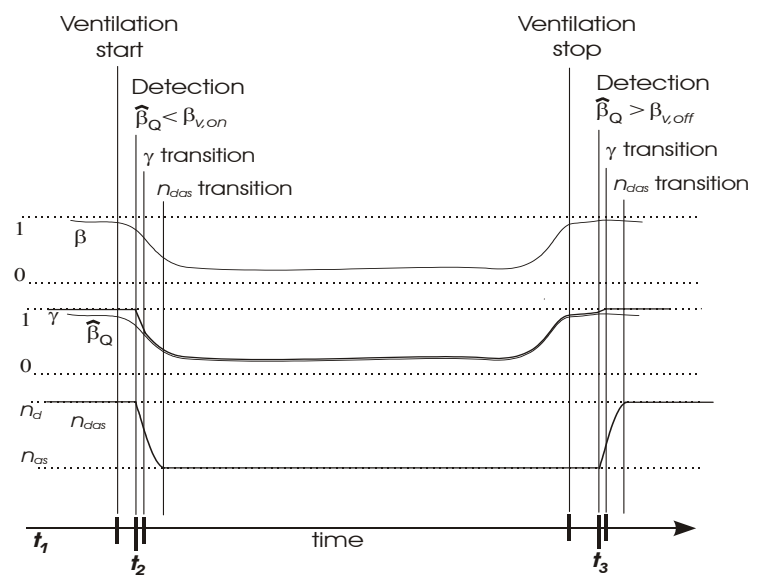

Fig. 2. Ventilation incident with detection and anti-spin control action.

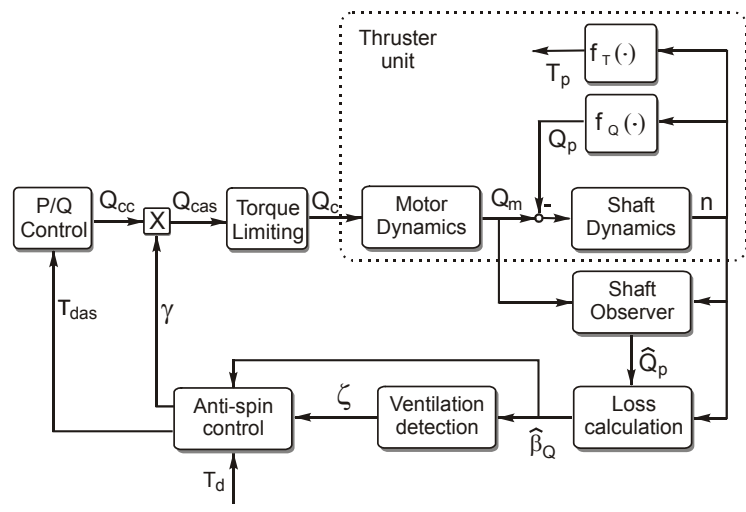

Fig. 3. The anti-spin control scheme, including combined power/torque controller, shaft observer, loss calculation, ventilation detection and antispin control.

$[L, B, T]=[80,18,5.6] m$ conducting DP operations in a sea state with significant wave height $H_{s}=5 \mathrm{~m}$. The thrust reference was $500 k N$. Fig. 4 shows the modelled ventilation loss $\beta_{Q}$ as a function of relative submergence $h / R$ and relative shaft speed $n / n_{\max }$, with the trace of a ventilating thruster with the anti-spin controller activated drawn as stars. The shaft speed of the propeller starts at $n / n_{\max } \approx 0.5$, and the ventilation incident starts when $h / R$ is reduced such that it falls over the edge for $h / R \approx$ 1.2. The loss of load torque leads to an increase in shaft speed until the ventilation is detected and the shaft speed is lowered to $n / n_{\max } \approx 0.35$. When ventilation is terminated, the shaft speed is increased to its original value.

Fig. 5 shows the propeller shaft speed, propeller thrust, propeller torque, motor torque and motor power for three different controllers:

- The combined power/torque controller with no anti-spin control action from (14) (thin line),

- The PID shaft speed controller from (11) (dotted line),

- The anti-spin controller with primary and secondary antispin control action from (15) and (22) (thick line).

The simulations illustrate the good properties of the proposed anti-spin controller. During ventilation the shaft speed PID controller has trouble with oscillations and engine racing, and the combined power/torque controller alone leads to severe engine racing. The anti-spin controller gives a less fluctuating and more responsive shaft speed than the PID controller, and reduces the shaft speed additionally during the ventilation situation. The result is more thrust, reduced wear and tear due to less peaks in the propeller load torque and motor torque, and less peaks in the motor power as the propeller re-enters the water. Fig. 6 shows details from the anti-spin controller for the same time series; actual torque loss $\beta_{Q}$, estimated torque loss $\hat{\beta}_{Q}$, torque modification $\gamma$, ventilation detection flag $\zeta$ and relative thrust reference $T_{d a s} / T_{d}$. The ventilation detection limits were set to $\beta_{v, o n}=0.6$ and $\beta_{v, o f f}=0.9$.

The anti-spin controller has been tested in simulations of varying environmental conditions, varying thrust references and varying levels of measurement noise, and showed good performance in all situations.

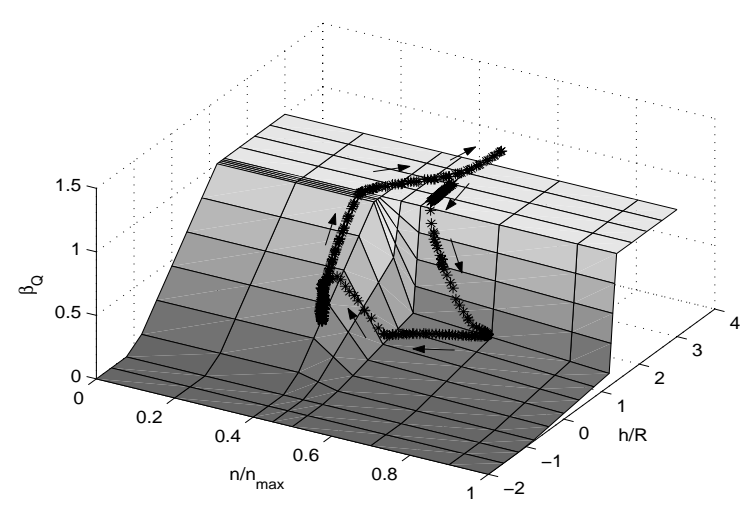

Fig. 4. Ventilation loss model with the trace of a ventilating propeller with anti-spin controller drawn as stars.

\section{CONCLUSION}

A thrust optimal anti-spin controller for marine propulsion systems in extreme conditions has been developed and tested by simulations based on experimental results. The controller utilized an estimate of the torque loss to detect ventilation incidents, which triggered an anti-spin control action. The primary anti-spin control action was to modify the output torque from the combined power/torque controller in order to take control of the propeller shaft speed. The secondary anti-spin control action was to reduce the thrust reference in order to lower the shaft speed to some optimal value. Simulations showed that the anti-spin controller gave improved thrust production, limited transients in the power system and reduced mechanical wear and tear when compared to conventional thruster control schemes during ventilation. It has earlier been shown that the combined controller had better performance than the shaft speed controller in moderate seas. The combined 

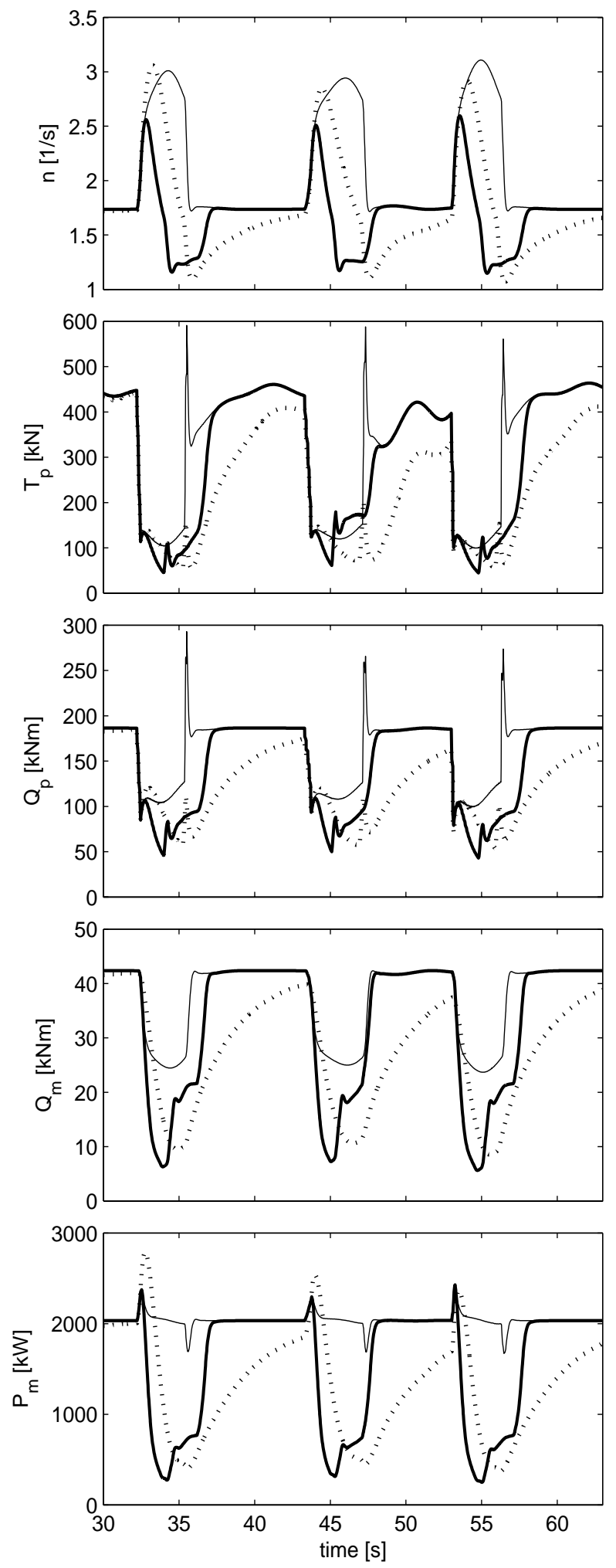

Fig. 5. Propeller shaft speed $n$, thrust $T_{p}$, load torque $Q_{p}$, motor torque $Q_{m}$ and motor power $P_{m}$ for the combined power/torque controller (thin line), the shaft speed PID controller (dotted line) and the anti-spin controller (thick line) during three ventilation incidents.

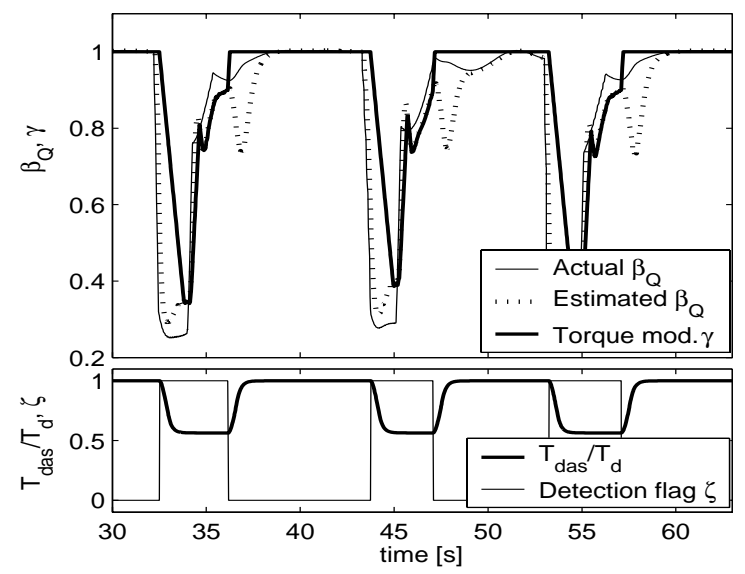

Fig. 6. Actual loss factor $\beta_{Q}$, estimated loss factor $\hat{\beta}_{Q}$, torque modification $\gamma$, loss detection flag $\zeta$ and relative thrust modification $T_{d a s} / T_{d}$ for the anti-spin controller during three ventilation incidents.

controller with anti-spin control action has thereby been shown to be preferable for all operating conditions.

\section{ACKNOWLEDGMENT}

This work has been carried out at the Centre for Ships and Ocean Structures (CESOS) at NTNU in cooperation with the research project on Energy-Efficient All-Electric Ship (EEAES). The Norwegian Research Council is acknowledged as the main sponsor of CESOS and EEAES.

\section{REFERENCES}

[1] K. J. Minsaas, H. J. Thon, and W. Kauczynski, "Estimation of required thruster capacity for operation of offshore vessels under severe weather conditions," in Proceedings of the $3^{\text {rd }}$ International Symposium on Practical Design of Ship and Mobile Units (PRADS'87), Trondheim, Norway, 1987, pp. 411-427.

[2] Ø. N. Smogeli, L. Aarseth, E. S. Overå, A. J. Sørensen, and K. J. Minsaas, "Anti-spin thruster control in extreme seas," in Proceedings of the $6^{\text {th }}$ IFAC Conference on Manoeuvring and Control of Marine Craft (MCMC'03), Girona, Spain, 2003, pp. 221-226.

[3] I. Haskara, U. Özgüner, and J. Winkelman, "Wheel slip control for antispin acceleration via dynamic spark advance," Control Engineering Practice, vol. 8, pp. 1135-1148, 2000.

[4] Ø. N. Smogeli, A. J. Sørensen, and T. I. Fossen, "Design of a hybrid power/torque thruster controller with thrust loss estimation," in Proceedings of the IFAC Conference on Control Applications in Marine Systems (CAMS'04), Ancona, Italy, 2004.

[5] T. I. Fossen and M. Blanke, "Nonlinear output feedback control of underwater vehicle propellers using feedback from estimated axial flow velocity," IEEE Journal of Oceanic Engineering, vol. 25, no. 2, pp. 241-255, 2000.

[6] L. L. Whitcomb and D. R. Yoerger, "Preliminary experiments in model-based thruster control for underwater vehicle positioning," IEEE Journal of Oceanic Engineering, vol. 24, no. 4, pp. 495-506, 1999.

[7] A. J. Sørensen, A. K. Ådnanes, T. I. Fossen, and J. P. Strand, "A new method of thruster control in positioning of ships based on power control," in Proceedings of the $4^{\text {th }}$ IFAC Conference on Manoeuvring and Control of Marine Craft (MCMC'97), Brijuni, Croatia, 1997, pp. 172-179.

[8] J. S. Carlton, Marine Propellers \& Propulsion. ButterworthHeinemann Ltd., 1994. 